\title{
CONTROLE DE MÁQUINAS DE INDUÇÃO PENTAFÁSICAS COM OTIMIZAÇÃO DO CAMPO NO ENTREFERRO
}

\author{
Luís A. Pereira* \\ lpereiraepucrs.br \\ Luís F. A. Pereira \\ lfpereiradece.ufrgs.br
}

\author{
César C. Scharlau ${ }^{\dagger}$ \\ cesarcsegmail.com \\ Sérgio Haffner ${ }^{\S}$ \\ haffner@ieee.org
}

*Programa de Pós-Graduação em Engenharia Elétrica

Pontifícia Universidade Católica do Rio Grande do Sul (PUCRS)

Av. Ipiranga, 6681, Partenon - Porto Alegre, RS, Brasil

${ }^{\dagger}$ Programa de Pós-Graduação em Engenharia de Automação e Sistemas

Universidade Federal de Santa Catarina (UFSC)

Caixa Postal 476, Trindade - Florianópolis, SC, Brasil

${ }^{\ddagger}$ Escola de Engenharia, Departamento de Engenharia Elétrica

Universidade Federal do Rio Grande do Sul (UFRGS)

Av. Osvaldo Aranha, 103, Centro - Porto Alegre, RS, Brasil

$\S$ Centro de Ciências Tecnológicas, Departamento de Engenharia Elétrica

Universidade do Estado de Santa Catarina (UDESC)

Campus Universitário Prof. Avelino Marcante, s/n, Bom Retiro - Joinville, SC, Brasil

\begin{abstract}
Five-phase Induction Machine Control with Optimized Air Gap Field

This paper presents the mathematical formulation of a dynamic model of five-phase induction machines. The model is based on symmetrical components of instantaneous value, decoupling the effects of the fundamental and the third harmonic air gap induction components. Through this model, two sets of dynamic equations are obtained, corresponding to the fundamental and the third harmonic state space representations of the machine equations. Simulation results are obtained employing a modified V/f control technique, which uses weighting factors for each harmonic component in order
\end{abstract}

\footnotetext{
Artigo submetido em 25/02/2009 (Id.: 00960)

Revisado em 14/05/2009

Aceito sob recomendação do Editor Associado Prof. Darizon Alves de An-
} drade to impose a trapezoidal induction waveform in the air gap of the machine. The simulation results are validated by practical tests.

KEYWORDS: AC motor drives, V/f control, Induction machines, Modeling.

\section{RESUMO}

Apresenta-se neste artigo a formulação matemática de um modelo dinâmico para máquinas de indução pentafásicas. $\mathrm{O}$ modelo apresentado é baseado em componentes simétricas de valor instantâneo, resultando no desacoplamento das componentes fundamental e de terceiro harmônico da indução no entreferro A partir deste modelo obtém-se dois conjuntos de equações que correspondem a representação em variáveis de estados do efeito de cada um dos componentes harmônicos nas equações da máquina. Resultados de simulação basea- 
dos em uma técnica de controle do tipo V/f, a qual utiliza fatores de ponderação para as componentes harmônicas a fim de impor de uma forma de onda de indução trapezoidal no entreferro da máquina, são comprovados experimentalmente validando o modelo proposto.

PALAVRAS-CHAVE: Motores CA, Controle V/f, Máquinas de Indução, Modelagem.

\section{LISTA DE SÍMBOLOS}

$m_{r}$ - número de fases do rotor;

$m_{s}$ - número de fases do estator;

$v_{k}^{s}$ - tensão aplicada na fase $k$ do estator;

$\widetilde{v}_{l}^{s}$ - tensão de sequência $l$ do estator;

$i_{k}^{s}$ - corrente da fase $k$ do estator;

$i_{k}^{r}$ - corrente da fase $k$ do rotor;

$\widetilde{i}_{l}^{s}$ - corrente de sequência $l$ do estator;

$\widetilde{i}_{l}^{r}$ - corrente de sequência $l$ do rotor;

$\widetilde{i}_{l}^{r s}$ - corrente de sequência $l$ do rotor referenciado ao estator;

$\omega_{e}$ - velocidade do rotor em graus elétricos/s;

$\omega_{m}$ - velocidade do rotor em graus mecânicos/s;

$S$ - escorregamento relativo do rotor;

$T_{e}$ - torque eletromagnético da máquina;

$T_{l}$ - torque de carga;

$J_{m}$ - coeficiente de atrito viscoso;

$B_{m}$ - coeficiente de amortecimento do conjunto motor e carga;

$\Psi_{k}^{s}$ - fluxo concatenado com a fase $k$ do estator;

$\Psi_{k}^{r}$ - fluxo concatenado com a fase $k$ do rotor;

$R_{k}^{s}$ - resistência da fase $k$ do estator;

$R_{k}^{r}$ - resistência da fase $k$ do rotor;

$R_{b}^{r}$ - resistência de uma barra do rotor;

$L_{\delta}^{s}$ - indutância de dispersão de uma fase do estator;

$L_{h}^{s}$ - indutância principal de uma fase do estator;

$L_{\delta}^{r}$ - indutância de dispersão de uma fase do rotor;

$L_{\delta b}^{r}$ - indutância de dispersão de uma barra do rotor;
$L_{h}^{r}$ - indutância principal de uma fase do rotor;

$\widehat{L}_{n}^{r s}$ - valor máximo da indutância mútua entre uma fase do estator e uma fase do rotor para o harmônico de ordem $n$;

$\phi$ - ângulo de deslocamento entre o eixo da fase 1 do estator e da fase 1 do rotor;

$p$ - número de pares de polos;

$\widetilde{R}^{s}$ - resistência de sequência do estator;

$\widetilde{L}_{j}^{s}$ - indutância de sequência $j$ do estator;

$\widetilde{R}_{j}^{r}$ - resistência de sequência $j$ do rotor;

$\widetilde{L}_{j}^{r}$ - indutância de sequência $j$ do rotor;

$\widehat{B}_{n}$ - amplitude da harmônica de ordem $n$ da indução produzida pelo estator;

$\widehat{I}_{n}$ - amplitude da harmônica de ordem $n$ da corrente do estator;

$\widehat{V}_{n}$ - amplitude da harmônica de ordem $n$ da tensão de fase do estator;

$B_{M}$ - indução máxima produzida por uma fase do estator;

$R$ - raio interno do estator;

$\gamma$ - ângulo entre duas ranhuras do estator;

$\mu_{0}$ - permeabilidade magnética do ar;

$\delta_{e}$ - entreferro equivalente;

$N_{s}$ - número de espiras por bobina do estator;

$\ell$ - comprimento axial do estator;

$\sigma_{p}$ - passo do enrolamento;

$\varphi$ - ângulo de defasagem entre duas fases consecutivas do estator;

$\varepsilon$ - ângulo de defasagem entre duas fases consecutivas fases do rotor.

Notação: “ " corresponde a componentes simétricas de valor instantâneo, “*" simboliza complexo conjugado e " $T$ " significa matriz transposta. 


\section{INTRODUÇÃO}

Devido às suas características de robustez, versatilidade e confiabilidade, a máquina de indução tem sido amplamente utilizada nas últimas décadas em várias aplicações. O desenvolvimento de novas topologias de inversores e sistemas de controle abriu uma gama de aplicações para a máquina de indução em áreas onde anteriormente a máquina de corrente contínua predominava.

Tradicionalmente, máquinas de indução com enrolamentos trifásicos têm sido empregadas em atuadores eletromecânicos. Isto se deve ao fato de que o sistema de alimentação industrial amplamente utilizado é também trifásico. Desta forma, a concepção das máquinas de indução é baseada em conceitos há muito tempo estabelecidos e tendo em vista as características da rede de alimentação industrial. No entanto, em acionamentos onde a máquina não é alimentada diretamente pela rede, não existe a exigência de um número específico de fases. Em alguns casos a escolha de um número maior de fases pode ser mais apropriada ou vantajosa (Toliyat, Rahimian e Lipo, 1991).

A análise do desempenho de diversas alternativas de enrolamentos para máquinas de indução alimentadas por inversores é abordado em (Toliyat, Lipo e White, 1991). Esse estudo conclui que uma máquina de cinco fases associada a um sistema de acionamento que obtenha uma distribuição de campo próxima da retangular apresenta um ganho de torque em comparação a uma máquina trifásica convencional. O mesmo estudo demostra que o ganho de torque não aumenta significativamente quando número de fases é maior que cinco. No entanto, a ênfase do estudo reside na análise da forma de onda de corrente do estator, não sendo analisados aspectos como a variação de perdas devido a correntes não senoidais ou mesmo na forma de onda da indução resultante no entreferro da máquina devido à ação conjunta das correntes do estator e do rotor. As vantagens da utilização de máquinas de indução pentafásicas sobre as máquinas trifásicas convencionais são também abordadas numa série de trabalhos publicados, como por exemplo em (Klingshirn, 1983; Ward e Harer, 1984; Singh, 2002; Jacobina et al., 2004; Levi et al., 2007).

A análise, projeto e aplicação de estratégias de controle para máquinas com número de fases maior que três exigem o estabelecimento de modelos matemáticos adequados que permitam avaliar o seu desempenho. Em (Pereira et al., 2006) é apresentado um modelo completo da máquina de indução pentafásica considerando harmônicos no campo do entreferro, os quais reconhecidamente desempenham um papel preponderante no desempenho de máquinas com número de fases maior que três. Neste modelo é obtido inicialmente um desacoplamento dos efeitos de cada harmônico de campo, de tal maneira que cada um dos componentes harmônicos é representado de forma independente, cada qual com o seu respectivo conjunto de equações dinâmicas. As equações que descrevem o comportamento dinâmico da máquina de indução pentafásica são representadas pelas componentes simétricas de valor instantâneo, resultando em um desacoplamento entre os componentes harmônicos da máquina. $\mathrm{O}$ desenvolvimento é particularizado para o caso em que são considerados somente o efeito da componente fundamental e do terceiro harmônico.

Com base nas equações correspondentes ao efeito de cada componente harmônico, é possível determinar estratégias de controle que melhorem o desempenho da máquina. Tradicionalmente, nas máquinas de indução trifásicas, a indução no entreferro possui uma distribuição senoidal, atingindo um valor máximo em uma região pequena. Isto resulta em baixo grau de aproveitamento do material ativo e uma densidade de potência e de torque menores. Para o caso das máquinas de indução pentafásicas, é possível obter um incremento do torque no eixo do motor quando a indução no entreferro da máquina possui uma forma de onda semelhante a retangular. Isto é obtido através da escolha de uma combinação apropriada dos componentes harmônicos do campo do entreferro. Scharlau et al. (2008) apresentam uma análise do desempenho de uma máquina de indução pentafásica, operando com uma estratégia de controle do tipo tensão/frequência, combinando os componentes fundamental e terceiro harmônico de corrente do estator, a fim de otimizar a distribuição da indução no entreferro da máquina.

Neste artigo é apresentada a extensão do modelo proposto por Pereira et al. (2006), considerando a representação em variáveis de estado dos componentes fundamental e terceiro harmônico. Com base neste modelo é apresentada uma estratégia de controle do tipo tensão/frequência, levando em consideração a combinação adequada dos componentes harmônicos de corrente para imposição da indução no entreferro com formato de onda próximo ao retangular. Resultados obtidos em simulação são comparados com resultados experimentais mostrando a validade do modelo dinâmico proposto para a máquina de indução pentafásica, bem como do método apresentado para determinação dos componentes harmônicos de corrente que resultam na indução desejada no entreferro da máquina.

\section{MODELO DA MÁQUINA DE INDUÇÃO PENTAFÁSICA}

Será apresentado nesta seção o modelo da máquina de indução pentafásica. O equacionamento apresentado foi particularizado para o protótipo de máquina pentafásica que foi utilizado para obtenção dos resultados experimentais, mas pode ser estendido para outros tipos de máquinas. Em máquinas 
de indução com rotor do tipo gaiola não existe um número de fases definido para o rotor. Para o protótipo utilizado foram consideradas no equacionamento do modelo da máquina um total de 15 fases para o rotor. Este número corresponde ao número de barras do rotor por polo.

Para a parte elétrica da máquina são consideradas as equações das tensões para o estator e para o rotor, conforme descritas a seguir:

$$
\begin{gathered}
{[v]^{s}=[R]^{s} \cdot[i]^{s}+\frac{d[\Psi]^{s}}{d t}} \\
{[0]=[R]^{r} \cdot[i]^{r}+\frac{d[\Psi]^{r}}{d t}}
\end{gathered}
$$

onde $[v]^{s}=\left[v_{1}^{s}, v_{2}^{s}, v_{3}^{s}, v_{4}^{s}, v_{5}^{s}\right]^{T}$ é o vetor de tensões do estator, $[i]^{s}=\left[i_{1}^{s}, i_{2}^{s}, i_{3}^{s}, i_{4}^{s}, i_{5}^{s}\right]^{T}$ é o vetor de correntes do estator, $[i]^{r}=\left[i_{1}^{r}, i_{2}^{r}, i_{3}^{r}, \cdots, i_{15}^{r}\right]^{T}$ é o vetor de correntes do rotor, $[\Psi]^{s}=\left[\Psi_{1}^{s}, \Psi_{2}^{s}, \Psi_{3}^{s}, \Psi_{4}^{s}, \Psi_{5}^{s}\right]^{T}$ é o vetor de fluxos concatenados do estator, $[\Psi]^{r}=\left[\Psi_{1}^{r}, \Psi_{2}^{r}, \Psi_{3}^{r}, \cdots, \Psi_{15}^{r}\right]^{T}$ é o vetor de fluxos concatenados do rotor, $[R]^{s}$ a matriz diagonal contendo as resistências próprias do estator e $[R]^{r}$ a matriz de resistências do rotor, ambas definidas a seguir:

$$
[R]^{s}=\operatorname{diag}\left[\begin{array}{lllll}
R_{1}^{s} & R_{2}^{s} & R_{3}^{s} & R_{4}^{s} & R_{5}^{s}
\end{array}\right]
$$

$$
\begin{aligned}
& {[R]^{r r}=} \\
& {\left[\begin{array}{cccccc}
R_{1}^{r} & -R_{b}^{r} & 0 & \cdots & 0 & -R_{b}^{r} \\
-R_{b}^{r} & R_{2}^{r} & -R_{b}^{r} & \cdots & 0 & 0 \\
0 & -R_{b}^{r} & R_{3}^{r} & \cdots & 0 & 0 \\
\vdots & \vdots & \vdots & \ddots & \vdots & \vdots \\
0 & 0 & 0 & \cdots & R_{14}^{r} & -R_{b}^{r} \\
-R_{b}^{r} & 0 & 0 & \cdots & -R_{b}^{r} & R_{15}^{r}
\end{array}\right]}
\end{aligned}
$$

As equações dos fluxos do estator e do rotor da máquina são apresentadas em (5) e (6).

$$
\begin{aligned}
& {[\Psi]^{s}=[L]^{s s} \cdot[i]^{s}+[L]^{s r} \cdot[i]^{r}} \\
& {[\Psi]^{r}=[L]^{r r} \cdot[i]^{r}+[L]^{r s} \cdot[i]^{s}}
\end{aligned}
$$

Substituindo as equações (5) e (6) em (1) e (2), obtém-se:

$$
[v]^{s}=[R]^{s} \cdot[i]^{s}+[L]^{s s} \cdot \frac{d[i]^{s}}{d t}+\frac{d\left\{[L]^{s r} \cdot[i]^{r}\right\}}{d t}
$$

$$
[0]=[R]^{r} \cdot[i]^{r}+[L]^{r r} \cdot \frac{d[i]^{r}}{d t}+\frac{d\left\{[L]^{r s} \cdot[i]^{s}\right\}}{d t}
$$

sendo $[L]^{s s}$ e $[L]^{r r}$ as matrizes de indutâncias próprias do estator e do rotor, $[L]^{s r}$ e $[L]^{r s}$ as matrizes de indutâncias mútuas entre o estator e o rotor, descritas na sequência:

$$
[L]^{s s}=[L]_{h}^{s s}+[L]_{\delta}^{s s}
$$

$$
\begin{aligned}
{[L]_{h}^{s s}=\frac{L_{h}^{s}}{9} \cdot } & {\left[\begin{array}{ccccc}
9 & 2 & -6 & -6 & 2 \\
2 & 9 & 2 & -6 & -6 \\
-6 & 2 & 9 & 2 & -6 \\
-6 & -6 & 2 & 9 & 2 \\
2 & -6 & -6 & 2 & 9
\end{array}\right] } \\
{[L]_{\delta}^{s s}=} & {\left[\begin{array}{ccccc}
L_{\delta}^{s} & 0 & 0 & 0 & 0 \\
0 & L_{\delta}^{s} & 0 & 0 & 0 \\
0 & 0 & L_{\delta}^{s} & 0 & 0 \\
0 & 0 & 0 & L_{\delta}^{s} & 0 \\
0 & 0 & 0 & 0 & L_{\delta}^{s}
\end{array}\right] }
\end{aligned}
$$

$$
[L]^{r r}=[L]_{h}^{r r}+[L]_{\delta}^{r r}
$$

$$
[L]_{h}^{r r}=\frac{L_{h}^{r}}{14} \cdot\left[\begin{array}{cccccc}
14 & -1 & -1 & \cdots & -1 & -1 \\
-1 & 14 & -1 & \cdots & -1 & -1 \\
-1 & -1 & 14 & \cdots & -1 & -1 \\
\vdots & \vdots & \vdots & \ddots & \vdots & \vdots \\
-1 & -1 & -1 & \cdots & 14 & -1 \\
-1 & -1 & -1 & \cdots & -1 & 14
\end{array}\right]
$$

$$
\begin{aligned}
& {[L]_{\delta}^{r r}=} \\
& {\left[\begin{array}{cccccc}
L_{\delta}^{r} & -L_{\delta b}^{r} & 0 & \cdots & 0 & -L_{\delta b}^{r} \\
-L_{\delta b}^{r} & L_{\delta}^{r} & -L_{\delta b}^{r} & \cdots & 0 & 0 \\
0 & -L_{b}^{r} & L_{\delta}^{r} & \cdots & 0 & 0 \\
\vdots & \vdots & \vdots & \ddots & \vdots & \vdots \\
0 & 0 & 0 & \cdots & L_{\delta}^{r} & -L_{\delta b}^{r} \\
-L_{\delta b}^{r} & 0 & 0 & \cdots & -L_{\delta b}^{r} & L_{\delta}^{r}
\end{array}\right]}
\end{aligned}
$$




$$
\begin{gathered}
{[S]=} \\
{\left[\begin{array}{ccccc}
1 & e^{j n(-\varphi)} & e^{j n(-2 \varphi)} & \cdots & e^{j n(-4 \varphi)} \\
e^{j n \varepsilon} & e^{j n(\varepsilon-\varphi)} & e^{j n(\varepsilon-2 \varphi)} & \cdots & e^{j n(\varepsilon-4 \varphi)} \\
e^{j n 2 \varepsilon} & e^{j n(2 \varepsilon-\varphi)} & e^{j n(2 \varepsilon-2 \varphi)} & \cdots & e^{j n(2 \varepsilon-4 \varphi)} \\
\vdots & \vdots & \vdots & \ddots & \vdots \\
e^{j n 14 \varepsilon} & e^{j n(14 \varepsilon-\varphi)} & e^{j n(14 \varepsilon-2 \varphi)} & \cdots & e^{j n(14 \varepsilon-4 \varphi)}
\end{array}\right]}
\end{gathered}
$$

$$
[L]^{s r}=\left\{[L]^{r s}\right\}^{T *}
$$

Para a parte mecânica, a equação que descreve o torque eletromagnético $\left(T_{e}\right)$ da máquina por ser expressa na forma matricial como:

$$
T_{e}=\frac{p}{2} \cdot\left[[i]^{s T} \mid[i]^{r T}\right]^{*} \cdot \frac{d}{d \phi}\left[\begin{array}{l|l}
{[L]^{s s}} & {[L]^{s r}} \\
\hline[L]^{r s} & {[L]^{r r}}
\end{array}\right] \cdot\left[\frac{[i]^{s}}{[i]^{r}}\right]
$$

As equações que descrevem o comportamento dinâmico da máquina (1-18), além de serem fortemente acopladas, não permitem avaliar a influência de cada harmônico em separado. Este sistema de equações pode ser consideravelmente simplificado através da introdução de transformações de coordenadas. Neste trabalho foi empregada a transformação para componentes simétricas de valor instantâneo, descrita em (White e Woodson, 1959). Esta transformação é aplicada diretamente aos valores instantâneos das tensões e das correntes, não sendo feita nenhuma restrição quanto à forma de onda das mesmas.

$$
\begin{aligned}
& {[v]^{s}=[P] \cdot[\widetilde{v}]^{s} \Leftrightarrow[\widetilde{v}]^{s}=[P]^{-1} \cdot[v]^{s}} \\
& {[i]^{s}=[P] \cdot[\widetilde{i}]^{s} \Leftrightarrow[\widetilde{i}]^{s}=[P]^{-1} \cdot[i]^{s}} \\
& {[i]^{r}=[Q] \cdot[\widetilde{i}]^{r} \Leftrightarrow[\widetilde{i}]^{r}=[Q]^{-1} \cdot[i]^{r}}
\end{aligned}
$$

onde $[\widetilde{v}]^{s}=\left[\begin{array}{lllll}\widetilde{v}_{0}^{s} & \widetilde{v}_{1}^{s} & \widetilde{v}_{2}^{s} & \widetilde{v}_{3}^{s} & \widetilde{v}_{4}^{s}\end{array}\right]^{T}$ é $\quad$ o vetor das componentes simétricas das tensões do estator, $[\tilde{i}]^{s}=\left[\begin{array}{lllll}\tilde{i}_{0}^{s} & \widetilde{i}_{1}^{s} & \widetilde{i}_{2}^{s} & \widetilde{i}_{3}^{s} & \widetilde{i}_{4}^{s}\end{array}\right]^{T}$ é o vetor das componentes simétricas das correntes do estator, $[\widetilde{i}]^{r}=\left[\begin{array}{lllll}\tilde{i}_{0}^{r} & \widetilde{i}_{1}^{r} & \widetilde{i}_{2}^{r} & \ldots & \widetilde{i}_{14}^{r}\end{array}\right]^{T}$ é o vetor das componentes simétricas das correntes do rotor. As componentes $i_{0}^{s}$ e $\widetilde{i}_{0}^{r}$ são as componentes de sequência zero da corrente do estator e do rotor e só tem valor diferente de zero quando a soma das correntes da máquina tiver um caminho de circulação.

Assumindo que a máquina está conectada em estrela e sem condutor neutro a componente de sequência zero $\widetilde{i}_{0}^{s}$ é nula, não sendo portanto necessário a consideração desta componente. Por consequência a componente de sequência zero do rotor $\widetilde{i}_{0}^{r}$ também é nula, uma vez que está concatenada com $\widetilde{i}_{0}^{s}$ do estator. As grandezas relacionadas com a componente fundamental são representadas pela componente simétrica de ordem um e quatro do estator e do rotor, as quais são complexas conjugadas uma da outra. Da mesma forma, as grandezas do terceiro harmônico são representadas pela componente simétrica de ordem dois e três, as quais também são complexas conjugadas uma da outra. Assim, para fins de equacionamento pode-se também considerar apenas uma componente simétrica para cada harmônico.

Para o estator, a transformação será definida pela matriz complexa (22), composta pelos autovetores da matriz $[L]^{s s}$,

$$
[P]=\frac{1}{\sqrt{5}} \cdot\left[\begin{array}{ccccc}
1 & 1 & 1 & 1 & 1 \\
1 & a^{-1} & a^{-2} & a^{-3} & a^{-4} \\
1 & a^{-2} & a^{-4} & a^{-6} & a^{-8} \\
1 & a^{-3} & a^{-6} & a^{-9} & a^{-12} \\
1 & a^{-4} & a^{-8} & a^{-12} & a^{-16}
\end{array}\right]
$$

sendo a constante complexa $a$ definida como:

$$
a=e^{j \frac{2 \cdot \pi}{m_{s}}}=e^{j \frac{2 \cdot \pi}{5}}
$$

A aplicação da matriz de transformação $[P]$ em $[L]^{s s}$ resulta em uma matriz diagonal $[\widetilde{L}]^{s s}$, eliminando o acoplamento que existia entre as fases através da indutância mútua. As indutâncias que aparecem na matriz $[\widetilde{L}]^{s s}$ são denominadas de indutâncias de sequência, conforme apresentado em (Pereira et al., 2006).

$$
[\widetilde{L}]^{s s}=[P]^{-1} \cdot[L]^{s s} \cdot[P]=\left[\begin{array}{ccccc}
\widetilde{L}_{0}^{s} & 0 & 0 & 0 & 0 \\
0 & \widetilde{L}_{1}^{s} & 0 & 0 & 0 \\
0 & 0 & \widetilde{L}_{2}^{s} & 0 & 0 \\
0 & 0 & 0 & \widetilde{L}_{2}^{s} & 0 \\
0 & 0 & 0 & 0 & \widetilde{L}_{1}^{s}
\end{array}\right]
$$

A mesma transformação é realizada para a matriz $[R]^{s}$, resultando em uma nova matriz diagonal $[\widetilde{R}]^{s}$, que neste caso é igual a $[R]^{s}$. 
A matriz de transformação utilizada nas equações do rotor da máquina (25) será composta pelos autovetores da matriz $[L]^{r r}$, ou seja

$$
[Q]=\frac{1}{\sqrt{15}} \cdot\left[\begin{array}{cccccc}
1 & 1 & 1 & 1 & \cdots & 1 \\
1 & b^{-1} & b^{-2} & b^{-3} & \cdots & b^{-14} \\
1 & b^{-2} & b^{-4} & b^{-6} & \cdots & b^{-28} \\
1 & b^{-3} & b^{-6} & b^{-9} & \cdots & b^{-42} \\
\vdots & \vdots & \vdots & \vdots & \ddots & \vdots \\
1 & b^{-14} & b^{-28} & b^{-42} & \cdots & b^{-196}
\end{array}\right]
$$

A constante complexa $b$ é definida como segue:

$$
b=e^{j \frac{2 \cdot \pi}{m_{r}}}=e^{j \frac{2 \cdot \pi}{15}}
$$

De forma semelhante a (24), a aplicação da matriz $[Q]$ nas matrizes de indutância e resistência do rotor permitem a obtenção das matrizes diagonais $[\widetilde{L}]^{r r}$ e $[\widetilde{R}]^{r}$, definidas em (Pereira et al., 2006).

A aplicação das transformações apresentadas em (19-21) ao conjunto de equações que descrevem a dinâmica da parte elétrica da máquina de indução pentafásica, descritas em (7) e (8), resultam em

$$
\begin{aligned}
& {[\widetilde{v}]^{s}=[\widetilde{R}]^{s} \cdot[\widetilde{i}]^{s}+[\widetilde{L}]^{s s} \cdot \frac{d[\widetilde{i}]^{s}}{d t}+\frac{d\left\{[\widetilde{L}]^{s r} \cdot[\widetilde{i}]^{r}\right\}}{d t}} \\
& {[0]=[\widetilde{R}]^{r} \cdot[\widetilde{i}]^{r}+[\widetilde{L}]^{r r} \cdot \frac{d[\widetilde{i}]^{r}}{d t}+\frac{d\left\{[\widetilde{L}]^{r s} \cdot[\widetilde{i}]^{s}\right\}}{d t}}
\end{aligned}
$$

onde

$$
\begin{gathered}
{[\widetilde{R}]^{s}=[P]^{-1} \cdot[R]^{s} \cdot[P]} \\
{[\widetilde{L}]^{s r}=[P]^{-1} \cdot[L]^{s r} \cdot[Q]} \\
{[\widetilde{R}]^{r}=[Q]^{-1} \cdot[R]^{r} \cdot[Q]} \\
{[\widetilde{L}]^{r r}=[Q]^{-1} \cdot[L]^{r r} \cdot[Q]}
\end{gathered}
$$

$$
[\widetilde{L}]^{r s}=[Q]^{-1} \cdot[L]^{r s} \cdot[P]=\left\{[\widetilde{L}]^{s r}\right\}^{T *}
$$

com a matriz $[\widetilde{L}]^{s s}$ já definida em (24).

Deve ser salientado que as transformações de coordenadas $[P]$ e $[Q]$ simplificam o sistema, mas de uma forma geral não diagonalizam as matrizes de indutâncias mútuas. Contudo, caso sejam consideradas apenas duas componentes harmônicas nas matrizes de indutâncias mútuas, as transformações de fato diagonalizam as matrizes de indutâncias mútuas. A dependência destas matrizes com a posição do rotor (e indiretamente com o tempo) contudo permanece. A introdução das variáveis em termos de componentes $q d$ para cada harmônico, apresentada mais adiante, permite que esta dependência também seja eliminada, resultando num sistema de equações diferenciais lineares.

As mesmas transformações são aplicadas na descrição do comportamento dinâmico da parte mecânica da máquina, representando-a como função das componentes simétricas de valor instantâneo. A partir de (18), obtém-se a seguinte expressão para o torque em termos das variáveis transformadas:

$$
\begin{aligned}
T_{e}= & \frac{p}{2} \cdot[\widetilde{i}]^{r T *} \cdot \frac{d[\widetilde{L}]^{r s}}{d \phi} \cdot[\widetilde{i}]^{s}+ \\
& \frac{p}{2} \cdot[\widetilde{i}]^{s T *} \cdot \frac{d[\widetilde{L}]^{s r}}{d \phi} \cdot[\widetilde{i}]^{r}
\end{aligned}
$$

As equações (27) e (28) podem ser reescritas apenas com as componentes simétricas de valor instantâneo do harmônico fundamental e do terceiro harmônico. Para isso, assume-se que a máquina está conectada em estrela, resultando como nulas as componentes de sequência zero do estator e do rotor, $\widetilde{i_{0}^{s}}$ e $\widetilde{i_{0}^{r}}$, respectivamente. Conforme apresentado em (Pereira et al., 2006), a harmônica fundamental está concatenada apenas com a primeira componente simétrica do estator e do rotor, obtendo-se as equações:

$$
\begin{gathered}
\widetilde{v}_{1}^{s}=\widetilde{R}^{s} \cdot \widetilde{i}_{1}^{s}+\widetilde{L}_{1}^{s} \cdot \frac{\widetilde{d i}_{1}^{s}}{d t}+M_{1} \cdot \frac{d}{d t}\left\{e^{j \cdot \phi} \cdot \widetilde{i}_{1}^{r}\right\} \\
0=\widetilde{R}_{1}^{r} \cdot \widetilde{i}_{1}^{r}+\widetilde{L}_{1}^{r} \cdot \frac{\widetilde{d i_{1}^{r}}}{d t}+M_{1} \cdot \frac{d}{d t}\left\{e^{-j \cdot \phi} \cdot \widetilde{i}_{1}^{s}\right\} \\
M_{1}=\frac{\sqrt{75}}{2} \cdot \widehat{L}_{1}^{r s}
\end{gathered}
$$


Da mesma forma, observa-se que o terceiro harmônico não exerce influência nas equações para a fundamental, sendo a equação portanto completamente independente. Para o terceiro harmônico de campo, semelhante ao conjunto de equações da fundamental, obtém-se as seguintes relações:

$$
\begin{gathered}
\widetilde{v}_{3}^{s}=\widetilde{R}^{s} \cdot \widetilde{i}_{3}^{s}+\widetilde{L}_{2}^{s} \cdot \frac{\widetilde{d i}_{3}^{s}}{d t}+M_{3} \cdot \frac{d}{d t}\left\{e^{j \cdot 3 \cdot \phi} \cdot \widetilde{i}_{3}^{r}\right\} \\
0=\widetilde{R}_{3}^{r} \cdot \widetilde{i}_{3}^{r}+\widetilde{L}_{3}^{r} \cdot \frac{\widetilde{d i_{3}^{r}}}{d t}+M_{3} \cdot \frac{d}{d t}\left\{e^{-j \cdot 3 \cdot \phi} \cdot \widetilde{i}_{3}^{s}\right\} \\
M_{3}=\frac{\sqrt{75}}{2} \cdot \widehat{L}_{3}^{r s}
\end{gathered}
$$

Conforme pode ser visto pelas expressões (38) e (39), os coeficientes das equações diferenciais da máquina são dependentes da posição do rotor, a qual em geral varia em função do tempo. Esta dependência pode ser eliminada aplicando-se uma segunda transformação de coordenadas a qual resultará em grandezas expressas nos eixos $q d$. Para tal, será considerado o referencial do estator, empregando-se as seguintes transformações para as variáveis do fundamental e de terceiro harmônico do rotor:

$$
\begin{gathered}
\widetilde{i}_{1}^{r s}=e^{j \cdot \phi} \cdot \widetilde{i}_{1}^{r} \\
\widetilde{i}_{3}^{r s}=e^{j \cdot 3 \cdot \phi} \cdot \widetilde{i}_{3}^{r}
\end{gathered}
$$

Utilizando as equações transformadas, é possível representar a máquina pentafásica através de uma máquina bifásica equivalente para cada harmônico nos eixos de quadratura $q d$, onde o eixo $q$ compreende a parte real e o eixo $d$ o negativo da parte imaginária. Por exemplo, a tensão do estator para o primeiro harmônico nos eixos $q d$ é descrita como $\widetilde{v}_{1}^{s}=\widetilde{v}_{1}^{s q}-j \widetilde{v}_{1}^{s d}$. Para as demais grandezas, a representação é efetuada de forma análoga.

As equações (43) e (44) descrevem o comportamento do motor para o componente fundamental:

$$
\begin{gathered}
{\left[\widetilde{v}_{1}\right]_{q d}^{s}=\widetilde{R}^{s} \cdot\left[\widetilde{i}_{1}\right]_{q d}^{s}+\widetilde{L}_{1}^{s} \cdot \frac{d}{d t}\left[\widetilde{i}_{1}\right]_{q d}^{s}+M_{1} \cdot \frac{d}{d t}\left[\widetilde{i}_{1}\right]_{q d}^{r s}} \\
0=\widetilde{R}_{1}^{r} \cdot\left[\widetilde{i}_{1}\right]_{q d}^{r s}+\widetilde{L}_{1}^{r} \cdot \frac{d}{d t}\left[\widetilde{i}_{1}\right]_{q d}^{r s}+ \\
M_{1} \cdot \frac{d}{d t}\left[\widetilde{i}_{1}\right]_{q d}^{s}+\omega_{e} \cdot\left[\widetilde{\Psi}_{1}\right]_{q d}^{r s}
\end{gathered}
$$

sendo

$$
\begin{aligned}
& {\left[\widetilde{v}_{1}\right]_{q d}^{s}=\left[\begin{array}{ll}
\widetilde{v}_{1}^{s q} & \widetilde{v}_{1}^{s d}
\end{array}\right]^{T}} \\
& {\left[\widetilde{i}_{1}\right]_{q d}^{s}=\left[\begin{array}{ll}
\widetilde{i}_{1}^{s q} & \widetilde{i}_{1}^{s d}
\end{array}\right]^{T}} \\
& {\left[\widetilde{i}_{1}\right]_{q d}^{r s}=\left[\begin{array}{ll}
\widetilde{i}_{1}^{r s q} & \widetilde{i}_{1}^{r s d}
\end{array}\right]^{T}} \\
& {\left[\widetilde{\Psi}_{1}\right]_{q d}^{r s}=\left[\begin{array}{ll}
\widetilde{\Psi}_{1}^{r s d} & -\widetilde{\Psi}_{1}^{r s q}
\end{array}\right]^{T}}
\end{aligned}
$$

De forma semelhante, as equações (45) e (46) são consideradas para o terceiro harmônico:

$$
\left[\widetilde{v}_{3}\right]_{q d}^{s}=\widetilde{R}^{s} \cdot\left[\widetilde{i}_{3}\right]_{q d}^{s}+\widetilde{L}_{2}^{s} \cdot \frac{d}{d t}\left[\widetilde{i}_{3}\right]_{q d}^{s}+M_{3} \cdot \frac{d}{d t}\left[\widetilde{i}_{3}\right]_{q d}^{r s}
$$

$$
\begin{aligned}
0= & \widetilde{R}_{3}^{r} \cdot\left[\widetilde{i}_{3}\right]_{q d}^{r s}+\widetilde{L}_{3}^{r} \cdot \frac{d}{d t}\left[\widetilde{i}_{3}\right]_{q d}^{r s}+ \\
& M_{3} \cdot \frac{d}{d t}\left[\widetilde{i}_{3}\right]_{q d}^{s}+3 \cdot \omega_{e} \cdot\left[\widetilde{\Psi}_{3}\right]_{q d}^{r s}
\end{aligned}
$$

sendo

$$
\begin{aligned}
& {\left[\widetilde{v}_{3}\right]_{q d}^{s}=\left[\begin{array}{ll}
\widetilde{v}_{3}^{s q} & \widetilde{v}_{3}^{s d}
\end{array}\right]^{T}} \\
& {\left[\widetilde{i}_{3}\right]_{q d}^{s}=\left[\begin{array}{ll}
\widetilde{i}_{3}^{s q} & \widetilde{i}_{3}^{s d}
\end{array}\right]^{T}} \\
& {\left[\widetilde{i}_{3}\right]_{q d}^{r s}=\left[\begin{array}{ll}
\widetilde{i}_{3}^{r s q} & \widetilde{i}_{3}^{r s d}
\end{array}\right]^{T}} \\
& {\left[\widetilde{\Psi}_{3}\right]_{q d}^{r s}=\left[\begin{array}{ll}
\widetilde{\Psi}_{3}^{r s d} & -\widetilde{\Psi}_{3}^{r s q}
\end{array}\right]^{T}}
\end{aligned}
$$

A equação do torque eletromagnético da máquina pode ser reescrita em termos das componentes $q d$ descritas pelas equações (43) a (46).

$$
\begin{aligned}
T_{e}= & 2 \cdot p \cdot M_{1} \cdot\left[\widetilde{i}_{1}^{s q} \cdot \widetilde{i}_{1}^{r d}-\widetilde{i}_{1}^{s d} \cdot \widetilde{i}_{1}^{r q}\right]+ \\
& 6 \cdot p \cdot M_{3} \cdot\left[\widetilde{i}_{3}^{s q} \cdot \widetilde{i}_{3}^{r d}-\widetilde{i}_{3}^{s d} \cdot \widetilde{i}_{3}^{r q}\right]
\end{aligned}
$$

O diagrama de blocos apresentado na Figura 1 ilustra a sequência de operações necessárias para a obtenção da representação do modelo dinâmico do motor em coordenadas $q d$, com desacoplamento entre as componentes relacionadas ao harmônico fundamental e ao terceiro harmônico. Nesta representação a tensão do estator da máquina $v^{s}$ é considerada como variável de entrada. Como variáveis de saída são consideradas a corrente do estator, a corrente do rotor e a 
velocidade angular mecânica do rotor, representadas respectivamente por $i^{s}, i^{r}$ e $\omega_{m}$. As equações (48) e (49) descrevem o comportamento dinâmico da parte mecânica da máquina.

$$
\begin{gathered}
-\frac{d \phi}{d t}=\omega_{e}=p \cdot \omega_{m} \\
T_{e}-T_{l}=\frac{1}{p} \cdot\left(J_{m} \cdot \frac{d^{2} \phi}{d t^{2}}+B_{m} \cdot \frac{d \phi}{d t}\right)
\end{gathered}
$$

Com base nas equações (43) a (46) que descrevem o comportamento dinâmico da parte elétrica da máquina para os componentes fundamental e terceiro harmônico, pode-se generalizar a representação na forma de espaço de estados para as componentes harmônicas de ordem $n$, sendo $n=1,3$ :

$$
\left\{\begin{aligned}
{[\dot{x}(t)]_{n} } & =\left[A\left(\omega_{e}\right)\right]_{n}[x(t)]_{n}+[B]_{n}[u(t)]_{n} \\
{[y(t)]_{n} } & =[C]_{n}[x(t)]_{n}
\end{aligned}\right.
$$

sendo:

$$
\begin{aligned}
& {[x(t)]_{n}=\left[\widetilde{i}^{s q} \widetilde{i}^{s d} \widetilde{\Psi}^{r q} \widetilde{\Psi}^{r d}\right]_{n}^{T}} \\
& {[u(t)]_{n}=\left[\widetilde{v}^{s q} \widetilde{v}^{s d}\right]_{n}^{T}} \\
& {\left[A\left(\omega_{e}\right)\right]_{n}=\left[\begin{array}{cccc}
a_{1 n} & 0 & a_{2 n} & a_{3 n} \cdot n \cdot \omega_{e} \\
0 & a_{1 n} & -a_{3 n} \cdot n \cdot \omega_{e} & a_{2 n} \\
a_{4 n} & 0 & a_{5 n} & -n \cdot \omega_{e} \\
0 & a_{4 n} & n \cdot \omega_{e} & a_{5 n}
\end{array}\right]} \\
& {[B]_{n}=\left[\begin{array}{cc}
b_{1 n} & 0 \\
0 & b_{1 n} \\
0 & 0 \\
0 & 0
\end{array}\right]} \\
& {[C]=\left[\begin{array}{llll}
1 & 0 & 0 & 0 \\
0 & 1 & 0 & 0
\end{array}\right]}
\end{aligned}
$$

As constantes que aparecem nas equações anteriores são definidas conforme segue:

$$
\begin{aligned}
a_{1 n} & =-\left(\frac{\widetilde{R}^{s} \cdot \widetilde{L}_{n}^{r}+M_{n}^{2} \cdot \tau_{r n}}{K_{n}}\right) \\
a_{2 n} & =\frac{M_{n} \cdot \tau_{r n}}{K_{n}} \\
a_{3 n} & =\frac{M_{n}}{K_{n}} \\
a_{4 n} & =M_{n} \cdot \tau_{r n} \\
a_{5 n} & =-\tau_{r n} \\
b_{1 n} & =\frac{\widetilde{L}_{n}^{r}}{K_{n}} \\
K_{n} & =\widetilde{L}_{n}^{s} \cdot \widetilde{L}_{n}^{r}-M_{n}^{2} \\
\tau_{r n} & =\frac{\widetilde{R}_{n}^{r}}{\widetilde{L}_{n}^{r}}
\end{aligned}
$$

\section{CONTROLE TENSÃO/FREQUÊNCIA PARA MÁQUINAS DE INDUÇÃO PEN- TAFÁSICAS}

Com base no equacionamento apresentado na seção anterior, esta seção aborda o controle da máquina utilizando os componentes fundamental e de terceiro harmônico de indução no entreferro tendo em vista manter uma forma de onda otimizada no entreferro.

Uma das técnicas de controle amplamente utilizada para máquinas de indução é a denominada tensão/frequência (V/f). $\mathrm{O}$ princípio de operação desta técnica consiste em manter a relação $\mathrm{V} / \mathrm{f}$ constante, independente do ponto de operação da máquina. Por ser de simples implementação, a técnica de controle V/f ainda é bastante utilizada em acionamentos de máquinas de indução onde é necessário velocidade variável (Munoz-Garcia et al., 1998).

Em máquinas de indução trifásicas, tradicionalmente a técnica V/f é implementada considerando que a indução no entreferro da máquina apresenta uma distribuição tipicamente senoidal. Desta forma, a indução atinge um valor máximo em uma região pequena, resultando em um baixo grau de aproveitamento do material ativo. Com base no modelo da máquina de indução pentafásica apresentado, é possível utilizar a técnica V/f, com imposição de uma indução no entreferro com formato de onda próximo ao trapezoidal, aproveitando melhor o material ativo e melhorando a densidade de potência e torque no eixo do motor. A imposição da indução com forma de onda trapezoidal no entreferro da máquina pentafásica é realizada através da composição dos componentes fundamental e terceiro harmônico. 


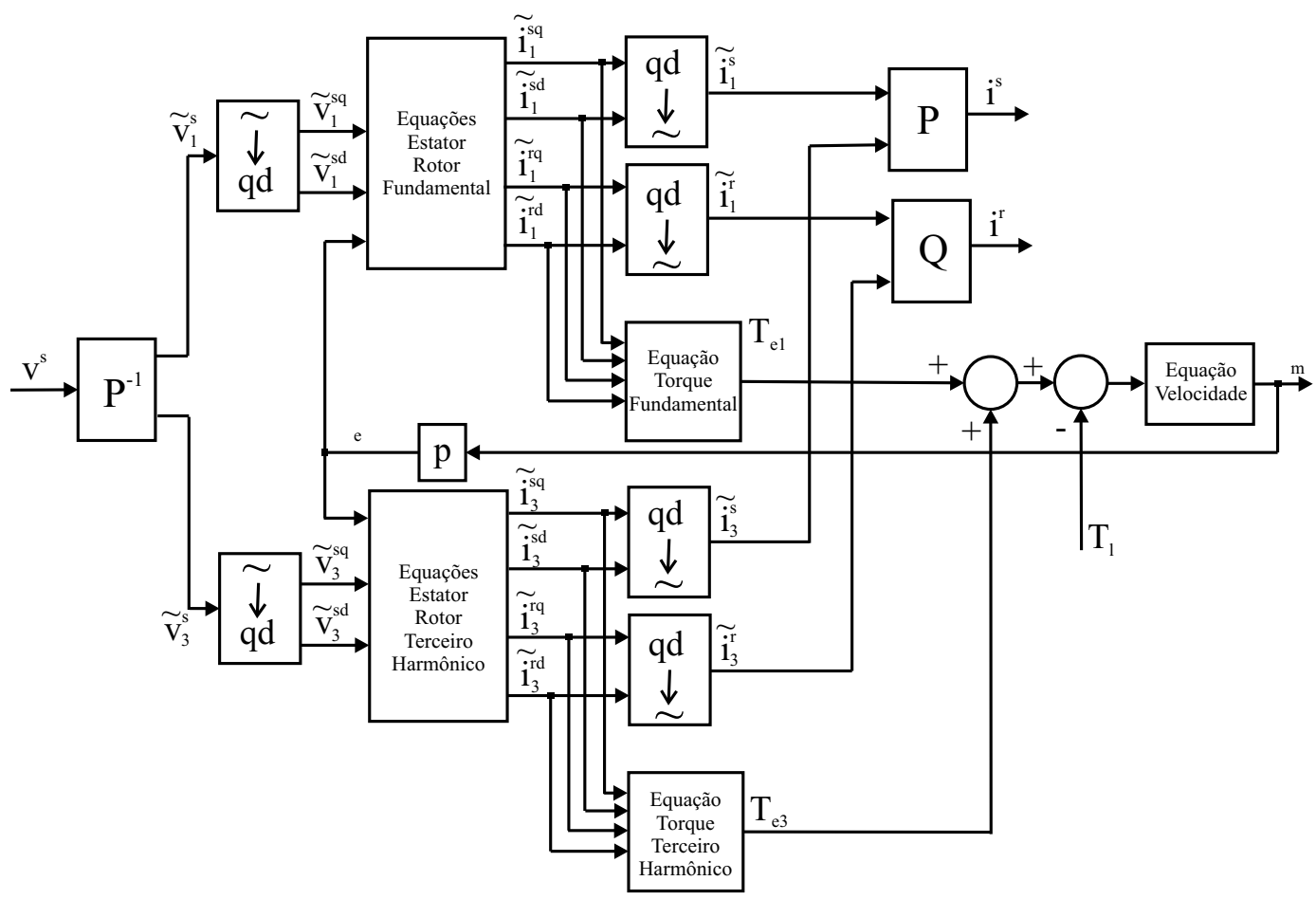

Figura 1: Diagrama de blocos do modelo da máquina de indução pentafásica.

A ideia de imposição de indução com formato de onda diferente do senoidal para máquinas pentafásicas já foi tema de trabalhos anteriores (Toliyat et al., 1998; Lyra e Lipo, 2002). Em (Xu et al., 2001) a imposição é feita utilizando uma estratégia de controle por orientação de campo, onde o módulo das componentes do terceiro harmônico da corrente do estator é determinado através de uma ponderação do módulo da componente fundamental da corrente do estator. Embora a ideia relacionada à ponderação das correntes das componentes fundamental e de terceiro harmônico seja adequada para a obtenção da forma de onda da indução trapezoidal no entreferro da máquina, os fatores de ponderação propostos em (Xu et al., 2001) não são adequados, pois tem por princípio a composição trapezoidal das correntes do estator da máquina, a qual não corresponde diretamente à indução no entreferro, conforme demonstrado no que segue. Será apresentado na sequência, tendo por base as equações do modelo da máquina apresentadas na seção anterior, o procedimento formal para obtenção da combinação adequada das componentes de corrente do estator da máquina que resultam em uma indução trapezoidal no entreferro da máquina.

Considerando a indução produzida no entreferro por uma fase do estator e desprezando os efeitos de saturação e distorção do campo produzidos pelas ranhuras do estator e do rotor, a densidade do campo sob um polo em um determinado instante de tempo pode ser aproximada pela forma de onda apresentada na Figura 2. Desta forma, a expressão matemática da indução no entreferro é dada pela equação que segue, onde foi considerando somente os efeitos da indução do componente fundamental e do terceiro harmônico, ou seja $n=1$ e $n=3$ :

$$
\begin{aligned}
B(\theta, t)= & \widehat{B}_{1} \sin \left(\theta+\omega_{e} t-\varphi_{1}\right)+ \\
& \widehat{B}_{3} \sin \left(3 \theta+3 \omega_{e} t-\varphi_{3}\right)
\end{aligned}
$$

sendo

$$
\widehat{B}_{1}=\frac{m_{s}}{2} \widehat{I}_{1}^{s} \widehat{W}_{1}
$$

e

$$
\widehat{B}_{3}=\frac{m_{s}}{2} \widehat{I}_{3}^{s} \widehat{W}_{3}
$$

O fator $\widehat{W}_{n}$, definido na equação (59), inclui os efeitos da distribuição dos enrolamentos sobre a superfície interna do estator e a permeância efetiva do entreferro.

$$
\widehat{W}_{n}=\frac{4}{\pi} \frac{1}{n} \cos \left(\frac{n \gamma}{2}\right) \frac{\mu_{0}}{\delta_{e}} N_{s}
$$




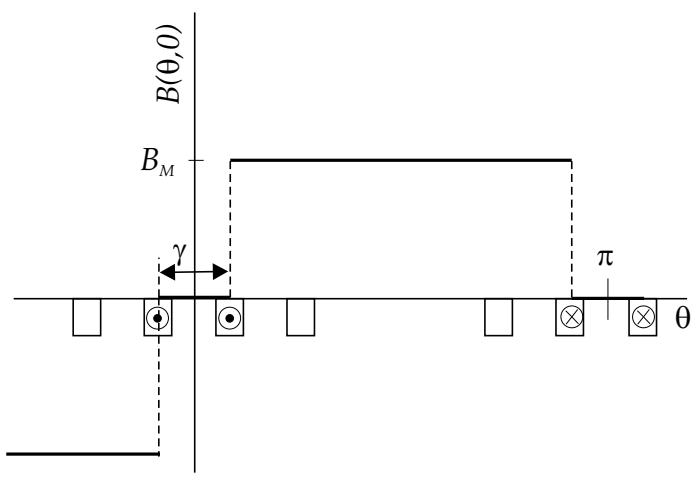

Figura 2: Indução no entreferro sob um polo produzida por uma fase do estator para uma máquina pentafásica com 2 ranhuras por fase e por polo e no instante de tempo $t=0$.

Desta forma, para uma determinada relação $\widehat{B}_{3} / \widehat{B}_{1}$ a relação correspondente entre as correntes $\widehat{I}_{3}$ e $\widehat{I}_{1}$ é

$$
\frac{\widehat{I}_{3}}{\widehat{I}_{1}}=\frac{\widehat{W}_{1} \widehat{B}_{3}}{\widehat{W}_{3} \widehat{B}_{1}}
$$

Para uma máquina de indução pentafásica com duas ranhuras por pólo e fase, conforme Figura 2, e enrolamento de passo inteiro, a relação entre $\widehat{W}_{3}$ e $\widehat{W}_{1}$ é aproximadamente igual a 3, resultando em:

$$
\frac{\widehat{I_{3}}}{\widehat{I}_{1}} \approx 3 \frac{\widehat{B}_{3}}{\widehat{B}_{1}}
$$

Na equação (61) é possível observar que a relação das amplitudes entre os componentes das correntes é diferente da relação das amplitudes entre os componentes da indução no entreferro, comprovando a afirmação de que a ponderação entre as correntes $\widehat{I}_{1}$ e $\widehat{I}_{3}$ que implicam uma imposição de corrente trapezoidal inviabiliza o formato de onda trapezoidal da indução no entreferro da máquina.

Com o objetivo de obter a forma da indução no entreferro, uma bobina de medição de passo inteiro foi instalada no estator, conforme visto na Figura 3. Considerando que a máquina possui 10 ranhuras por polo, a primeira parte da bobina de medição está localizada na ranhura 1 e a segunda parte na ranhura 11. O fluxo resultante que cruza a área da bobina de medição é calculado considerando que os lados da bobina se encontrem em posições arbitrárias $\alpha$ e $\alpha+\pi$, conforme dado a seguir:

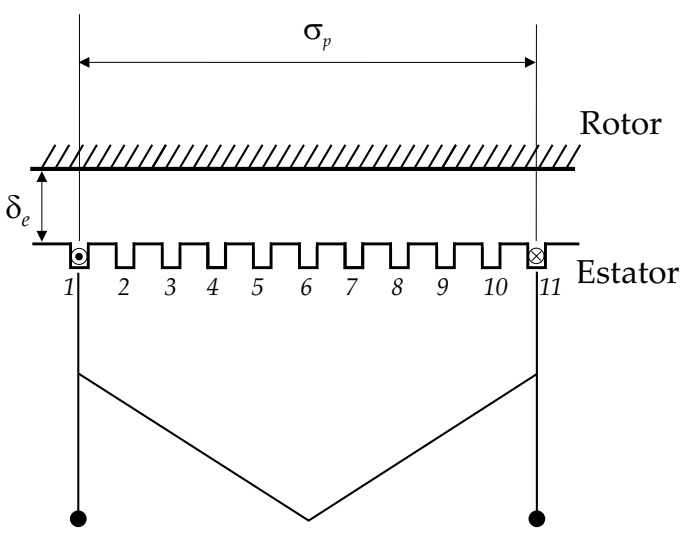

Figura 3: Bobina de medição com passo inteiro.

$$
\begin{aligned}
\Phi(\alpha, t)= & \frac{-2 \ell R}{p}\left[\widehat{B}_{1} \cos \left(\alpha+\omega_{e} t-\varphi_{1}\right)+\right. \\
& \left.\frac{\widehat{B}_{3}}{3} \cos \left(3 \alpha+3 \omega_{e} t-\varphi_{3}\right)\right]
\end{aligned}
$$

onde $\alpha$ descreve a posição da primeira parte da bobina de medição no sistema de coordenadas fixo do estator.

A tensão $v(\alpha, t)$ induzida nos terminais da bobina de medição é obtida pela derivada no tempo de (62), conforme apresentado na sequência.

$$
\begin{gathered}
v(\alpha, t)=-\frac{d \Phi(\alpha, t)}{d t} \\
v(\alpha, t)=\frac{2 \omega_{e} \ell R}{p}\left[\widehat{B}_{1} \sin \left(\alpha+\omega_{e} t-\varphi_{1}\right)+\right. \\
\left.\widehat{B}_{3} \sin \left(3 \alpha+3 \omega_{e} t-\varphi_{3}\right)\right]
\end{gathered}
$$

Comparando as equações (56) e (63) verifica-se que a forma de onda da tensão induzida nos terminais da bobina de medida apresenta o mesmo formato da indução produzida no entreferro pelas componentes de corrente do fundamental e do terceiro harmônico. A diferença entre as equações está localizada somente no fator de multiplicação, pois os termos entre colchetes são iguais aos da equação (56).

Portanto, a distribuição no tempo da tensão induzida $v(\alpha, t)$ corresponde exatamente a distribuição espacial da indução $B(\theta, t)$. Essa equivalência é explicada considerando em um instante arbitrário de tempo, por exemplo $t=0$, e uma posição arbitrária do estator, por exemplo $\alpha=0$, nas equações (56) e (63) conforme demonstrado a seguir. 


$$
\begin{aligned}
B(\theta, 0)= & \widehat{B}_{1} \sin \left(\theta-\varphi_{1}\right)+ \\
& \widehat{B}_{3} \sin \left(3 \theta-\varphi_{3}\right) \\
v(0, t)= & K_{u}\left[\widehat{B}_{1} \sin \left(\omega_{e} t-\varphi_{1}\right)+\right. \\
& \left.\widehat{B}_{3} \sin \left(3 \omega_{e} t-\varphi_{3}\right)\right]
\end{aligned}
$$

sendo

$$
K_{u}=\frac{2 \omega_{e} \ell R}{p} .
$$

Observa-se que (56) e (65) possuem a mesma forma de onda, distinguindo-se somente pela amplitude, que corresponde ao fator $K_{u}$. Adicionalmente, $B(\theta, t)$ representa uma onda girante com um formato fixo e uma velocidade rotacional $\omega_{e}$ em qualquer instante de tempo. Para simplificar a simbologia, no restante do artigo a indução $B(\theta, t)$ será referida como $B(\theta)$ e a tensão induzida $v(\alpha, t) \operatorname{como} v(t)$.

A tensão induzida representada por (63) considera somente o efeito das correntes do estator para o caso da máquina sem carga. Quando a máquina está operando com carga o campo resultante no entreferro é produzido não somente pelas correntes do estator, mas também pelas correntes do rotor. O efeito das correntes do rotor no campo do entreferro pode ser incluído na equação (63) multiplicando-se as correntes do estator pelos seguintes fatores complexos:

$$
\begin{gathered}
\bar{K}_{1}^{r}=1-\frac{m_{r}}{2} \frac{m_{s}}{2} \frac{\left(\widehat{L}_{1}^{s r}\right)^{2}}{\widetilde{L}_{1}^{s}} \frac{j \omega_{e}}{\left[\frac{\widetilde{R}_{1}^{r}}{S}+j \omega_{e} \widetilde{L}_{1}^{r}\right]} \\
\bar{K}_{1}^{r}=\widehat{K}_{1}^{r} \angle \varphi_{k 1} \\
\bar{K}_{3}^{r}=1-\frac{m_{r}}{2} \frac{m_{s}}{2} \frac{\left(\widehat{L}_{3}^{s r}\right)^{2}}{\widetilde{L}_{3}^{s}} \frac{j 3 \omega_{e}}{\left[\frac{\widetilde{R}_{3}^{r}}{S}+j 3 \omega_{e} \widetilde{L}_{3}^{r}\right]} \\
\bar{K}_{3}^{r}=\widehat{K}_{3}^{r} \angle \varphi_{k 3}
\end{gathered}
$$

Desta forma, a expressão em regime permanente da tensão induzida na bobina de medição considerando as correntes do rotor é dada por:
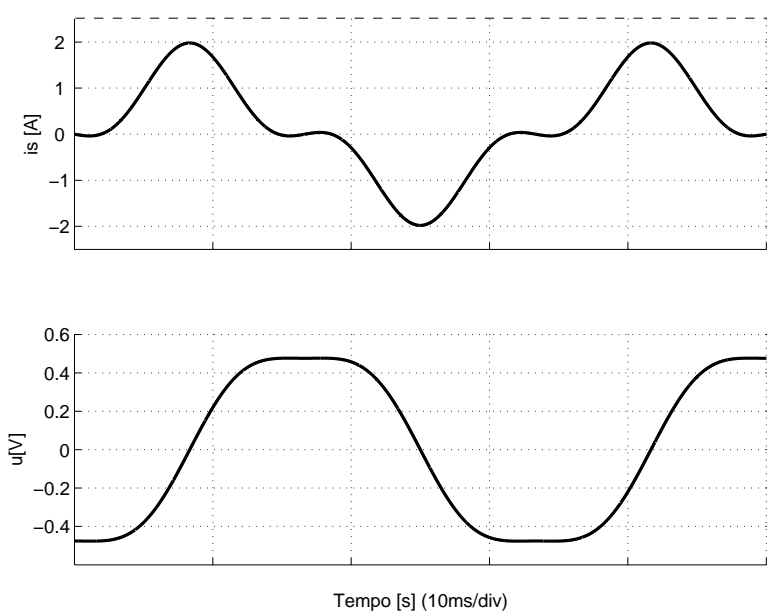

Figura 4: Corrente do estator e a respectiva indução no entreferro da máquina.

$$
\begin{array}{r}
v(t)=K_{u}\left[\widehat{B}_{1} \widehat{K}_{1}^{r} \sin \left(\omega_{e} t-\varphi_{1}+\varphi_{k 1}\right)+\right. \\
\left.\widehat{B}_{3} \widehat{K}_{3}^{r} \sin \left(3 \omega_{e} t-\varphi_{3}+\varphi_{k 3}\right)\right]
\end{array}
$$

De forma a avaliar a influência da relação entre as componentes fundamental e terceiro harmônico da corrente do estator na forma de onda da indução no entreferro da máquina, diversas simulações foram efetuadas utilizando a equação (65). Nesta equação é possível observar que a relação entre as amplitudes das componentes de corrente fundamental e de terceiro harmônico é diferente da relação entre as amplitudes das formas de onda dos componentes da indução. Além disso, a defasagem entre as fases não é igual. Esse fato é ilustrado na Figura 4, onde a amplitude do terceiro harmônico da corrente do estator possui amplitude de $40 \%$ do componente fundamental, enquanto a indução correspondente do terceiro harmônico possui amplitude de $14 \%$ do componente fundamental. A defasagem da corrente é de 180 graus enquanto a defasagem da indução é de 0 graus. Isto comprova que a onda da indução no entreferro não segue o mesmo formato da onda da corrente no estator.

Para impor uma indução com formato próximo ao trapezoidal, a forma de onda da corrente deve ser cuidadosamente projetada e baseada nas expressões matemáticas que definem a indução no entreferro descritas em (71). O problema em questão é a obtenção da relação entre $\widehat{B}_{1}$ e $\widehat{B}_{3}$ que resulte na forma de onda desejada. Este problema foi resolvido em (Scharlau et al., 2008) através de um procedimento de otimização cujo objetivo era o de minimizar a amplitude da oscilação resultante na crista de uma onda trapezoidal composta genericamente por duas ondas senoidais representando, res- 
pectivamente, as componentes fundamental e terceiro harmônico. A relação obtida como resultado deste procedimento é de $\widehat{B}_{3} / \widehat{B}_{1}=0,137$. Com base na equação (61) e na relação $\widehat{B}_{3} / \widehat{B}_{1}$, obtém-se a proporção das amplitudes das correntes do fundamental e do terceiro harmônico que deverão ser impostas no estator da máquina para que o formato da indução seja trapezoidal, ou seja $\widehat{I}_{3} / \widehat{I}_{1} \approx 0,411$.

Como no controle do tipo V/f é necessária a imposição das tensões, utilizam-se as equações extraídas dos modelos em regime permanente da máquina das componentes fundamental e de terceiro harmônico, apresentadas em (72) e (73).

$$
\begin{aligned}
& \widehat{V}_{1}=\widehat{I}_{1}\left|R^{s}+j \omega_{e} \widetilde{L}_{1}^{s}\right| \\
& \widehat{V}_{3}=\widehat{I}_{3}\left|R^{s}+j 3 \omega_{e} \widetilde{L}_{3}^{s}\right|
\end{aligned}
$$

\section{SIMULAÇÕES E RESULTADOS EXPE- RIMENTAIS}

Com o objetivo de validar e verificar a performance da topologia de controle proposta foi utilizada uma plataforma de testes para máquinas elétricas, descrita em (Spiller et al., 2002). A Figura 5 apresenta o diagrama de blocos dos equipamentos utilizados na plataforma. O programa Matlab/Simulink encontra-se instalado no computador, juntamente com as ferramentas de tempo real, simulação e controle. O protótipo da máquina de indução pentafásica foi construído utilizando-se a carcaça de uma máquina trifásica de construção fechada de 4 polos e $3 / 4$ cv. Os dados básicos da máquina podem ser vistos na Tabela 1 e os principais parâmetros são apresentados na Tabela 2, sendo que os valores se referem a um par de polos. Durante os testes, a indução no entreferro foi obtida através da tensão induzida na bobina de medição. Os sinais provenientes da máquina, como a velocidade do rotor, correntes e tensões do estator são adquiridos através de uma placa de interface e aquisição desenvolvida para a plataforma de testes. O inversor pentafásico foi implementado utilizando dois inversores trifásicos e encontra-se conectados ao protótipo da máquina pentafásica. A estratégia de modulação utilizada foi o PWM senoidal com frequência de chaveamento de $20 \mathrm{kHz}$. Foram utilizados filtros do tipo passa-baixas de segunda ordem na aquisição das correntes e das tensões. A plataforma também disponibiliza para os testes uma carga programável implementada utilizando um motor de corrente contínua operando nos quatro quadrantes.

As Figuras 6 e 7 apresentam a simulação e os resultados experimentais da estratégia de controle proposta operando com duas condições de carga distintas, porém com as mesmas relações V/f para cada harmônico.

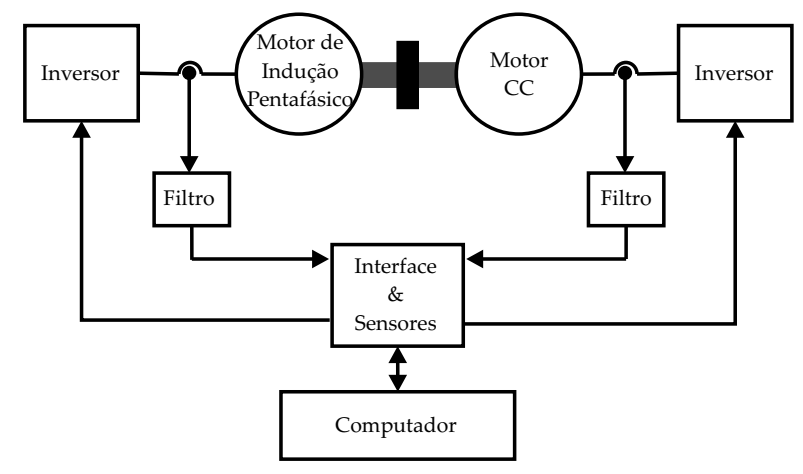

Figura 5: Plataforma de testes para máquinas elétricas.

Tabela 1: Dados do Protótipo.

\begin{tabular}{|l|c|}
\hline diâmetro externo do estator & $130,3 \mathrm{~mm}$ \\
\hline diâmetro interno do estator $(D)$ & $80,0 \mathrm{~mm}$ \\
\hline comprimento axial $(\ell)$ & $64,0 \mathrm{~mm}$ \\
\hline entreferro corrigido $\left(\delta_{e}\right)$ & $0,48 \mathrm{~mm}$ \\
\hline passo do enrolamento $\left(\sigma_{p}\right)$ & 10 ranhuras \\
\hline número de espiras/bobina $\left(N_{s}\right)$ & 90 \\
\hline número de ranhuras do estator & 40 \\
\hline número de ranhuras do rotor & 30 \\
\hline número de polos & 4 \\
\hline
\end{tabular}

A Figura 6 está relacionada aos ensaios efetuados sem carga. Na Figura 6(a) são apresentadas as curvas da tensão faseneutro do estator e na Figura 6(b) as medições de corrente do estator. Pode-se observar que a ponderação dos valores de tensão do fundamental e do terceiro harmônico resultam na imposição de uma indução no entreferro com o formato de onda desejado, como visto na Figura 6(c). Neste teste verifica-se também uma diferença de fase entre os componentes fundamental e terceiro harmônico da corrente do estator em aproximadamente 180 graus, enquanto a diferença de fase na tensão do estator e na indução no entreferro é próxima de zero. Comparando-se os resultados obtidos em simulação com os experimentais, verifica-se que ambos estão em bom acordo, especialmente para a as curvas da indução no entreferro da máquina. É importante salientar que os efeitos de saturação e distorção de campo provocados pelas ranhuras do estator e do rotor afetam os valores das indutâncias de uma forma bastante difícil de determinar utilizando métodos analíticos. Estes efeitos podem podem ser considerados de forma aproximada por meio de fatores conhecidos na literatura como o fator de saturação e fator de Carter quando da determinação das indutâncias de forma analítica. No presente artigo as indutâncias principais foram determi- 
Tabela 2: Parâmetros do protótipo.

\begin{tabular}{|c|c||c|c|}
\hline Parâmetro & Valor & Parâmetro & Valor \\
\hline$R^{s}$ & $3,48 \Omega$ & $L_{\delta}^{s}$ & $4 \mathrm{mH}$ \\
\hline$L_{h}^{r}$ & $0,79 \mu \mathrm{H}$ & $L_{h}^{s}$ & $74,5 \mathrm{mH}$ \\
\hline$\widetilde{R}_{1}^{r}$ & $8,10 \mu \Omega$ & $\widetilde{R}_{3}^{r}$ & $42,50 \mu \Omega$ \\
\hline$\widehat{L}_{1}^{s r}$ & $76,4 \mu \mathrm{H}$ & $\widehat{L}_{3}^{s r}$ & $21,9 \mu \mathrm{H}$ \\
\hline$\widetilde{L}_{1}^{s}$ & $168,9 \mathrm{mH}$ & $\widetilde{L}_{3}^{s}$ & $21,0 \mathrm{mH}$ \\
\hline$\widetilde{L}_{1}^{r}$ & $0,71 \mu \mathrm{H}$ & $\widetilde{L}_{3}^{r}$ & $0,92 \mu \mathrm{H}$ \\
\hline
\end{tabular}

nadas por meio do método de elementos finitos em duas dimensões para a condição de saturação nominal da máquina. Por outro lado, as indutâncias de disperesão foram em parte determinadas por métodos analíticos, como por exemplo a dispersão relativa à cabeça das bobinas. Desta forma os efeitos de saturação e distorção foram incluídos nos parâmetros da máquina. Como o modelo da máquina é um modelo linear, os valores das indutâncias foram incorporados ao modelo como valores constantes, o que introduz um certo erro ao modelo. As discrepâncias entre os resultados experimentais e de simulação são mais visíviveis nas curvas de corrente que dependem fortemente dos valores das indutâncias, os quais podem variar com a condição de carga e saturação da máquina.

Outros ensaios foram efetuados considerando a máquina operando com carga nominal (2,7 N.m). A simulação e os resultados experimentais são apresentados na Figura 7. Verifica-se que aplicando uma tensão no estator com o mesmo valor do ensaio sem carga também foi obtida uma indução no entreferro com formado de onda trapezoidal. No entanto, o formato de onda da corrente apresentou alterações. Comparando-se as Figuras 6(b) e 7(b) observa-se uma mudança na diferença de fase entre os componentes harmônicos que compõem a corrente do estator. A mudança no formato de onda da corrente do estator pode ser explicada considerando que os circuitos equivalentes que correspondem a cada um dos componentes harmônicos possuem valores de impedância de entrada diferentes. Estes valores de impedância também se alteram de forma distinta conforme a condição de carga, resultando em diferentes formas de onda da corrente do estator para cada condição de carga. A mudança no formato de onda da corrente também pode ser explicada considerando que o campo resultante no entreferro com a máquina operando sob carga é produzido não somente pelas correntes do estator mas também pelas correntes do rotor, conforme estabelecido nas equações (67) a (71). A relação entre as correntes do estator e do rotor muda conforme a carga para manter o formato de onda da indução no entreferro inalte-

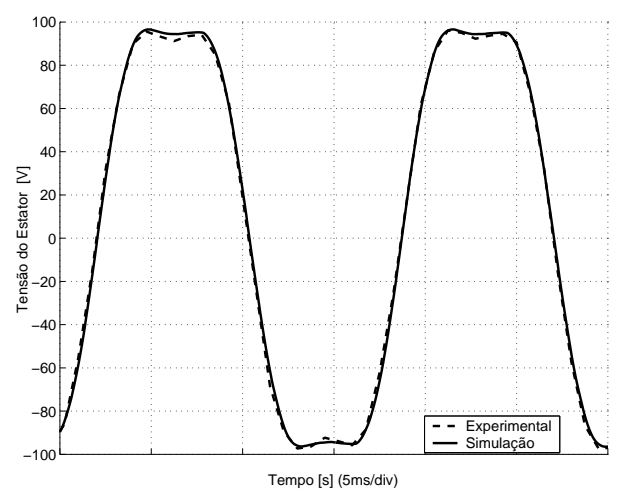

(a) Tensão do Estator.

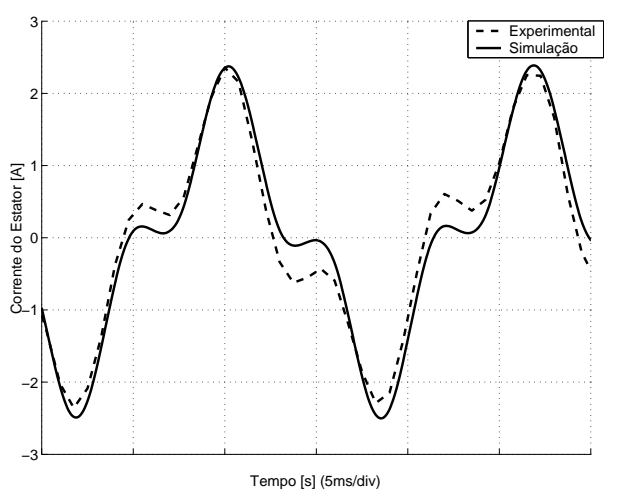

(b) Corrente do Estator.

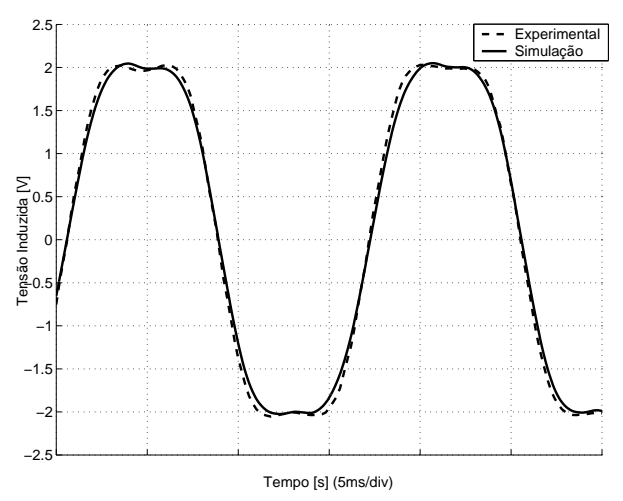

(c) Tensão Induzida.

Figura 6: Simulação e resultados experimentais da estratégia de controle operando sem carga.

rado. Desta forma, uma indução trapezoidal no entreferro é produzida com correntes do estator com formas de onda diferentes da trapezoidal. Os resultados deste ensaio também apresentam boa similaridade entre as curvas obtidas em simulação e experimentalmente.

De forma a realizar uma comparação entre a estratégia de controle proposta e o método V/f convencional foram efetu- 


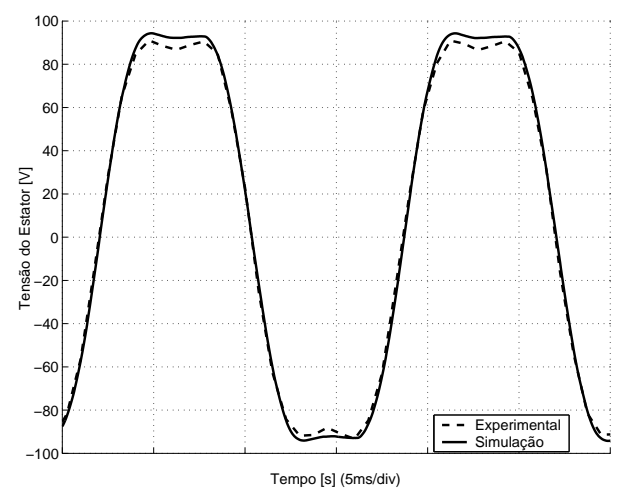

(a) Tensão do Estator.

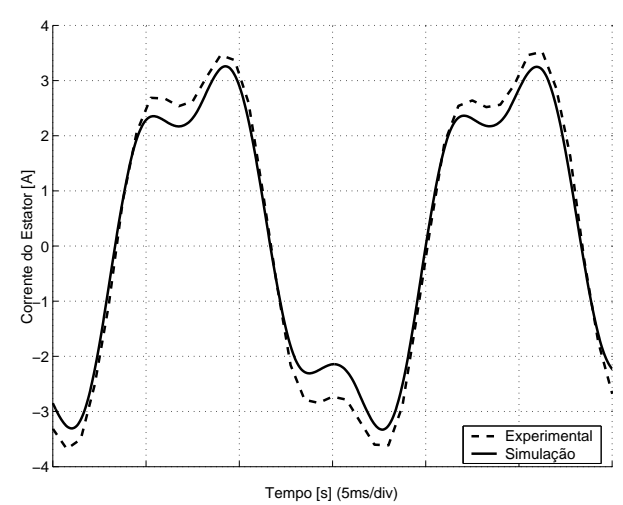

(b) Corrente do Estator.

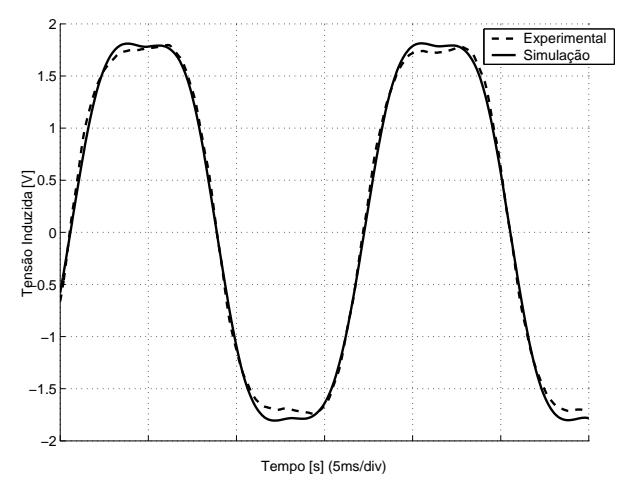

(c) Tensão Induzida.

Figura 7: Simulação e resultados experimentais da estratégia de controle operando com carga nominal.

ados testes mantendo o nível máximo de indução no entreferro da máquina. No esquema proposto este nível foi estabelecido para a forma de onda trapezoidal e na estratégia de controle tradicional o mesmo nível foi estabelecido para a forma de onda senoidal, conforme apresentado na Figura 8. O desempenho dinâmico da máquina operando com as duas estratégias de controle foi comparado levando-se em conta a partida da máquina a vazio com a aplicação de uma carga de
2.7 N.m após quatro segundos de operação. A Figura 9 apresenta as curvas de velocidade angular do rotor para as duas situações, observando-se que para a estratégia de controle proposta a resposta de velocidade foi mais rápida, apresentando também uma redução no erro em regime permanente de operação após a inclusão da carga.

A melhora na resposta dinâmica e o aumento do torque produzido se deve basicamente a dois fatores. O primeiro é o torque adicional produzido pelo terceiro harmônico, o qual é possível de ser produzido em máquinas pentáfasicas, devido ao fato que o terceiro harmônico de corrente poder circular mesmo com a máquina conectada em estrela sem neutro. $\mathrm{O}$ segundo fator é o aumento do torque produzido pelo componente fundamental, o qual foi possível devido a inclusão do terceiro harmônico. Mantendo-se a mesma indução máxima no entreferro, pode-se aumentar a componente fundamental da indução.

Deve-se salientar que a inclusão do terceiro harmônico altera a distribuição de campo não apenas no entreferro, mas em todas as demais partes da máquina (dentes do rotor e estator, coroa do estator e rotor, etc). Desta forma, as perdas também se alteram, em especial as perdas no ferro, as quais dependem não apenas do valor máximo da indução mas também da sua distribuição. Conforme mostrado em (Hsu et al., 1989) as perdas no ferro podem tanto aumentar quanto diminuir, dependendo como as mesmas se dividem entre perdas por histerese e por correntes parasitas. A proporção entre os dois tipos de perdas também varia em função do valor máximo da indução e da frequência (velocidade do rotor). A avaliação das perdas é, portanto, uma questão complexa e que está além do escopo do presente artigo. Nas análises apresentadas foi assumido que as perdas no ferro permanecem aproximadamente constantes com a inclusão do terceiro harmônico nos níveis em que ele foi inserido.

\section{CONCLUSÕES}

Este artigo apresentou a descrição do modelo matemático de uma máquina de indução pentafásica, levando em consideração o efeito dos componentes fundamental e terceiro harmônico. Para a obtenção do modelo foi utilizada a representação em componentes simétricas de valor instantâneo, que possibilitou o desacoplamento do efeito de cada um dos componentes harmônicos. Desta forma, foi possível obter dois conjuntos de equações dinâmicas da máquina, um deles associado ao fundamental e o outro relacionado ao terceiro harmônico. A partir do equacionamento obtido foram apresentadas as equações dinâmicas da máquina de indução pentafásica na forma de espaço de estados. Esta forma de representação facilita o desenvolvimento de novas estratégias de controle.

A partir do modelo proposto, foi desenvolvida uma aplicação 


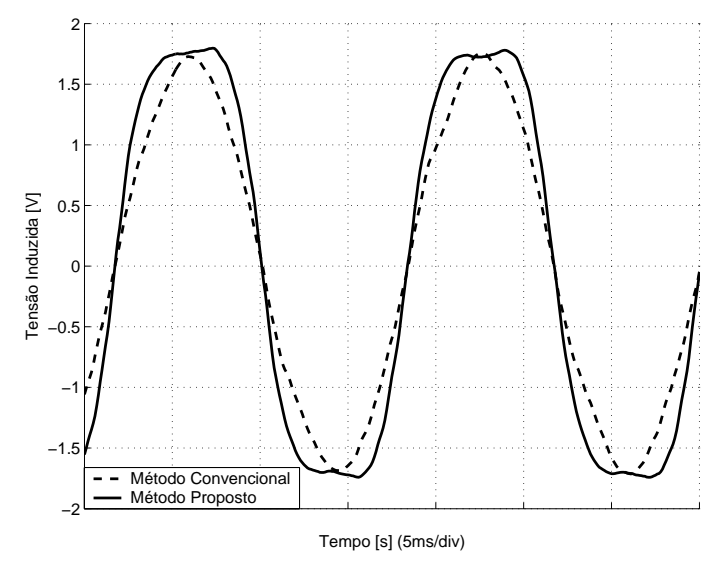

Figura 8: Tensão induzida na bobina de testes (indução no entreferro) utilizando a estratégia de controle proposta comparada com o método V/f convencional.

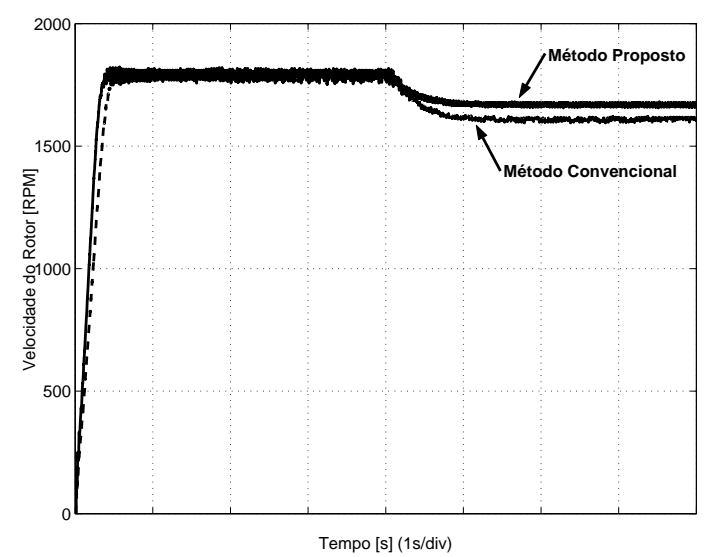

Figura 9: Desempenho dinâmico da estratégia de controle proposta comparada com o método V/f convencional.

específica de controle baseado na técnica V/f. O objetivo da estratégia de controle proposta foi a utilização da composição harmônica das correntes para obtenção de uma indução no entreferro com formato de onda trapezoidal, o qual permite um aumento de torque e uma melhor utilização do material ativo. Foram realizadas simulações e ensaios experimentais que validaram o modelo proposto. Através dos resultados obtidos, observa-se que a inclusão do terceiro harmônico produz uma parcela adicional de torque, implicando uma melhoria das respostas transitória e de regime permanente da máquina. $\mathrm{O}$ torque adicional provém basicamente do torque produzido pelo terceiro harmônico e do acréscimo do torque produzido pela componente fundamental, a qual pôde ser aumentada mantendo-se o mesmo nível de indução máxima no entreferro.
O modelo por variáveis de estado proposto neste artigo permitirá o desenvolvimento de trabalhos futuros relacionados ao projeto de observadores de estados e sua utilização em estratégias de controle por orientação do campo para os componentes harmônicos.

\section{AGRADECIMENTOS}

Este trabalho foi parcialmente financiado pelo $\mathrm{CNPq}$ através da concessão de bolsas de produtividade em pesquisa associadas aos processos 309389/2008-9 e 306280/2008-6.

\section{REFERÊNCIAS}

Hsu, J., Woodson, H. e Liou, S.-S. (1989). Experimental study of harmonic-flux effects in ferromagnetic materials, IEEE Trans. Magn. 25(3): 461-468.

Jacobina, C., de Azevedo, C., Lima, A. e de Souza Ribeiro, L. (2004). Online estimation of the stator resistance and leakage inductance of a four-phase induction machine drive, IEEE Trans. Power Electron. 19(1): 10-15.

Klingshirn, E. A. (1983). High phase order induction motors (parts i and ii), IEEE Trans. Power App. Syst. PAS102: 47-59.

Levi, E., Bojoi, R., Profumo, F. e Toliyat, H. (2007). Multiphase induction motor drives - A technology status review, IET - Electric Power Applications 1(4): 489-516.

Lyra, R. e Lipo, T. (2002). Six-phase induction machine with third harmonic current injection, Proc. Electrimacs.

Munoz-Garcia, A., Lipo, T. A. e Novotny, D. W. (1998). A new induction motor v/f control method capable of high-performance regulation at low speeds, IEEE Trans. Ind. Applicat. 34(4): 813-821.

Pereira, L. A., Scharlau, C. C., Pereira, L. F. A. e Haffner, J. F. (2006). General model of a five-phase induction machine allowing for harmonics in the air gap field, IEEE Trans. Energy Conversion 21(4): 891-899.

Scharlau, C. C., Pereira, L. F. A., Pereira, L. A. e Haffner, S. (2008). Performance of a five-phase induction machine with optimized air gap field under open loop $V / f$ control, IEEE Trans. Energy Conversion 23(4): 10461056.

Singh, G. (2002). Multi-phase induction machine drive research-a survey, Electric Power Systems Research 61(2): 139-147.

Spiller, P. A., Haffner, J. F. e Pereira, L. F. A. (2002). Aplicação em tempo real de ferramentas de simulação e im- 
plementação de técnicas de controle de máquinas de indução em ambiente Matlab/Simulink, XIV Congresso Brasileiro de Automática.

Toliyat, H. A., Lipo, T. e White, J. C. (1991). Analysis of a concentrated winding induction machine for adjustable speed drive applications (parts i and ii), IEEE Trans. Energy Conversion 6(4): 679-692.

Toliyat, H. A., Rahimian, M. M. e Lipo, T. A. (1991). dq modeling of five-phase synchronous reluctance machines including third harmonic of air gap mmf, IEEE Industry Applications Society Annual Meeting, pp. 231-237.

Toliyat, H., Waikar, S. e Lipo, T. (1998). Analysis and simulation of five-phase synchronous reluctancemachines including third harmonic of airgap mmf, IEEE Trans. Applicat. Ind. 34(2): 332-339.

Ward, E. E. e Harer, H. (1984). Preliminary investigation of an inverter-fed 5-phase induction motor, Proc. Inst. Elect. Eng., Vol. 116, pp. 980-984.

White, D. C. e Woodson, H. H. (1959). Electromechanical Energy Conversion, John Wiley and Sons, New York, NY.

Xu, H., Toliyat, H. A. e Petersen, L. J. (2001). Rotor field oriented control of five-phase induction motor with combined fundamental and third harmonic currents, Applied Power Electronics Conference and Exposition - APEC 2001, Vol. 1, pp. 392-398. 